\title{
Reducing Darcy coefficient by using drag reduction methods in open-channel flows: Effect on discharge capacity and potential application to mitigate river flooding impact
}

\author{
Emmanuel Mignot ${ }^{1,}$, , Nicolas Riviere ${ }^{1}$, Arnaud Lefevre $^{2}$ and Bernard Quillien $^{2}$ \\ ${ }^{1}$ Univ Lyon, INSA Lyon, CNRS, LMFA UMR5509, F-69621 Villeurbanne, France \\ ${ }^{2}$ SNF SAS, Rue Adrienne Bolland, ZAC de Milieux, 42163 Andrézieux Cédex, France
}

\begin{abstract}
Drag reduction by polymer addition is a common strategy used to minimize friction losses in pipe flows but has not been tested in river flows. Present paper then aims at measuring backwater curves and velocity profiles within smooth and rough bed flume configurations to assess the capabilities of such polymer addition to decrease the water depth with regards to the use of plain water and thus increase the channel conveyance. The inclusion of a limited amount of polymers proves to be able to reduce the typical Darcy-Weisbach friction coefficient with regards to plain water by a factor 2 in smooth bed conditions and a factor 1.5 in rough bed conditions. Moreover, the vertical profiles of streamwise velocity appear to be hardly affected by the addition of such polymers. Whether such drag reduction would still be effective in real watercourses remains unknown and would now require field experiments at larger scale.
\end{abstract}

\section{Introduction}

Discovered by Toms in 1948 [1], the drag reduction effect by polymer addition is a common strategy used to minimize friction losses when carrying fluids over long distances. It allows to dramatically reducing the wall friction and thus the linear head losses by up to $80 \%$. The origin of this phenomenon lies in the interactions between the turbulent structures of the flow and the polymer macromolecules added to the fluid [2]. In a fully-developed flow over a smooth bed, the velocity profile in the boundary layer is modified by the polymers. With clear-water, the velocity profile in the near-wall regions is composed of the laminar viscous sub-layer very close to the wall $\left(y u_{*} / \mathrm{v}<10\right.$ with $y$ the distance to the wall, $u_{*}$ the so-called friction velocity and $v$ the kinematic viscosity of the liquid) and the log law slightly further away $\left(y u_{*} / v>50\right)$. When adding polymers, the so-called "elastic zone" places itself in between these two zones, for $y^{+}>15$ ([3], [4]) within which the velocity profile follows a logarithmic law with a slope that depends on the polymer concentration and is not even universal. [5] also investigated the possibility of polymers to reduce the drag over rough surfaces. He observed that for walls with large enough roughness elements,

\footnotetext{
* Corresponding author: emmanuel.mignot@insa-lyon.fr
} 
the wall roughness tends to decrease the drag reduction process. The author could establish useful asymptotic regimes of drag reduction as a function of the relative roughness, flow and polymeric parameters. His main conclusions are that, for a water-polymer mixture, the effect of wall roughness begins to be effective for $k_{s}^{+}>12$ with $k_{s}^{+}=k_{s} u * / v$ (compared to $k_{s}^{+}>5$ in pure water). Then, for $12<k_{s}^{+}<50$, an "effectively smooth regime" is observed. Finally, for $k_{s}^{+}>50$, the drag reduction decreases compared to the smooth regime up to the experimental limit of $k_{s}^{+} \sim 150$.

Most works report inclusion of drag reduction polymers in pressurized pipe or channel flows. However, a few tests have also been performed in open-channel flows, especially within sewer systems. [6] reported the use of such polymers in the sewer network of the city of Whistler (Canada) during the 2010 Winter Olympic games. Their figures 6-7, for instance, show a strong water depth decrease and velocity increase in a sewer during a "high flow polymer dosing trial". Use of polymers in urban flows is made possible by the Materials Safety Data Sheet (MSDS) information for anionic polymers that indicates that the chemicals are non-toxic at limited dose concentrations [6].

Apart from these engineering applications in sewer networks, very little attention seems to have been devoted to drag reduction in open-channel flows. The aim of the present work is thus to fill this gap and to estimate the drag reduction efficiency in openchannel flows over smooth or rough beds. The dedicated experimental open-channel flume and measurement techniques are first introduced along with a description of the tested polymer additives. Then, the flow characteristics (water depths and velocity profiles) are measured with and without polymers and compared to each other i) under smooth bed and ii) rough bed conditions.

\section{Experimental set-up}

SNF is a leading company in drag reduction additives, mostly dedicated to Oil \& Gas extraction. Polymers from this company are tested herein in open-channel configurations. The additives are water-soluble polyacrylamide polymers (PAM or PAAM), commonly used as flocculants in water treatment processes (drinking water and waste water), as described by [7]. It is verified that the water/polymers mixture (up to 30ppm) remains Newtonian and its viscosity is increases by about $15 \%$ compared to plain water and also reveals that the flow in the flume tends to decrease this viscosity increment due to shearing effect on the polymers.

The experiments presented below took place in the laboratory of SNF at Andrezieux in France. A specific flume was designed and installed to investigate the effect of drag reduction additives on open-channel flows in different conditions. The flume is smooth, made of fiberglass and epoxy resin with a typical roughness size of $1.6 \mu \mathrm{m}$, of $b=12 \mathrm{~cm}$ wide rectangular cross-section with a constant streamwise slope of $0.011 \%$. The inlet discharge is controlled and measured in the upstream feeding system using valves and an electromagnetic flow meter. At the downstream outlet, the flume ends with a free-fall, imposing a critical flow regime before the water is evacuated outside the laboratory.

Measurements used in this paper consist of water depths and flow velocities. Water depths are measured using an ultrasonic radar sensor probe (Vegapuls 64 from VEGA) from above the flume. The 3 components of the flow velocity are measured at high sampling frequency locally using an acoustic Doppler velocimeter (16MHz ADV from Sontek). To improve the signal backscattering, tracers are added to the water and it was verified that these tracers do not affect the impact of the polymers on the drag reduction. 


\section{Smooth bed experiments}

This section aims at characterizing the drag reduction due to additives diluted in the water in an open-channel flow with smooth walls. To do so, five upstream discharges (see Table 1) are set, one after the other, under steady state conditions without or with different controlled concentrations of polymers. Keeping a critical downstream depth, a total of 21 backwater curves are measured. Figure 1 exhibits the measured backwater curves over the downstream 100-meters for a given selected discharge without and with four polymer concentrations. Fig. 1 confirms i) that the use of polymers decreases the water depths and, in return, increases the flow velocity, over the whole measured reach, ii) that the water depth reduction increases with the increasing polymer concentration and iii) that the drag reduction does not increase anymore as the polymer concentration exceeds 20 to $30 \mathrm{ppm}$, in agreement with the maximum drag reduction asymptote (MDRA) [3].

The backwater curves are fitted using a constant averaged Darcy friction coefficients $\lambda$ and Fig. 1 confirms that these curves fit the measured backwater curves with high fidelity both with plain water (leading to $\lambda \sim 0.017$, in agreement with Moody diagram) and with water/polymer mixtures (leading to lower Darcy coefficients). In the end, for a given discharge, the difference in water depth with / without polymers leads to a very limited difference in Reynolds number but to a strong decrease of friction coefficient: $\lambda$ is reduced by a factor of about 2 for all discharges (not shown here). When plotting these results on the well-known Moody diagram, $\lambda$ appears to be much lower than what can be obtained by the hydraulically-smooth curve. To conclude, the drag reduction in open-channel flows by adding polymers exceeds that of using a perfectly smooth wall, in agreement with pipe flow cases, reported for instance by [8].

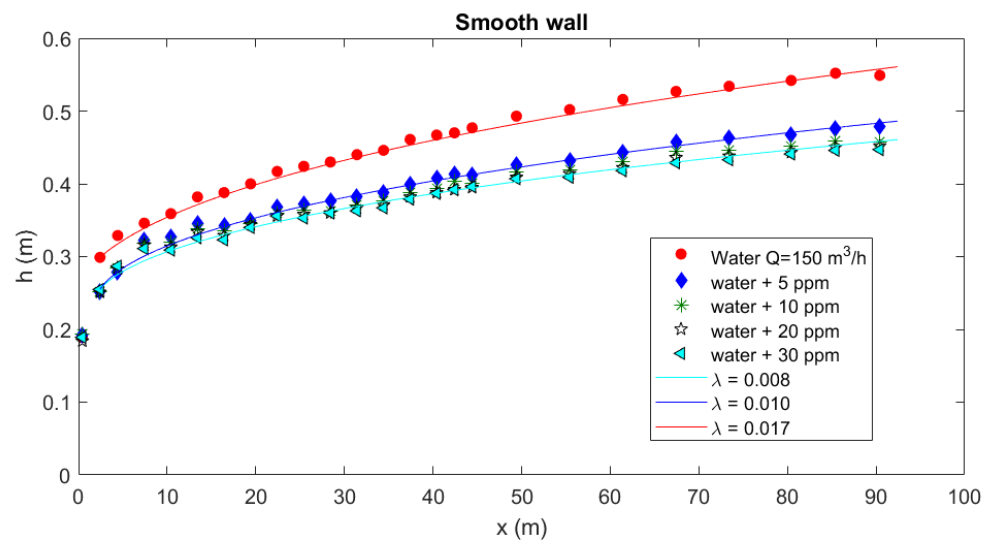

Fig. 1. Backwater curves measured (symbols) and fitted with a constant Darcy friction coefficient $\lambda$ (lines) for a discharge $Q=150 \mathrm{~m}^{3} / \mathrm{h}$ with different concentrations of additives in the smooth bed configuration, with $x$ the streamwise axis oriented towards upstream and $x=0$ the abscissa of the downstream free-fall (the water flows from right to left).

We now compare the vertical profiles of mean streamwise velocity measured in the smooth open-channel flow with and without the additives. Velocity profiles are measured over the lower $70 \%$ of the flow section using the ADV at a fixed location $(x=6.87 \mathrm{~m})$ for two discharges $\left(Q=50\right.$ and $\left.100 \mathrm{~m}^{3} / \mathrm{h}\right)$ without polymers and with a concentration equal to 20 ppm (see Fig.2). It should be reminded that varying the discharge and additives concentration for the four experiments while measuring at the same location in the flume leads to four different water depths and bulk velocities; Fig.2 thus compares profiles made non-dimensional using the water depth $h$ and bulk velocity $U_{b}$. It appears that the four velocity profiles are quite similar to each other and exhibit the expected global shape: a log 
region near the bed and a quite constant velocity profile further up in the water column in the bulk. In Fig. $2, b / h$ ranges from 0.7 to 1.3 (with $b$ the channel width and $h$ the local water depth) and these profiles are indeed highly similar to typical velocity profiles of narrow and deep open-channel flows in the literature, as shown, for instance by [9] with $b / h \sim 1.1$. Unfortunately, the ADV is not able to measure the details of the velocity profiles in the near-bed region to highlight the transitions between viscous, elastic (with additives) and logarithmic sub-layers. Such measurements would require technics such as PIV (as for [4]) which could not be implemented in the present flume as the lateral walls are not transparent.

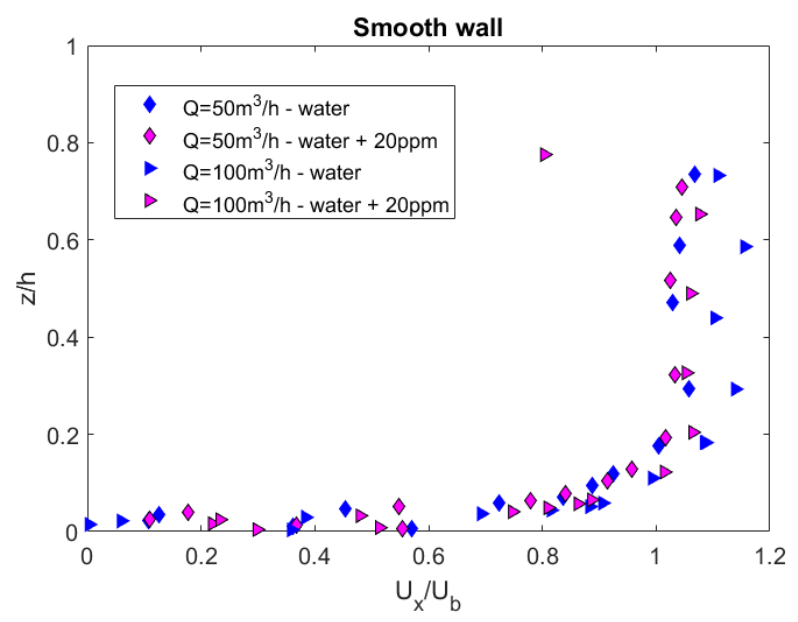

Fig. 2. Vertical profiles of non-dimensional mean streamwise velocities at a given channel abscissa for two discharges, with and without polymers.

\section{Rough bed experiments}

The previous section showed that the additives reduce the resisting wall friction in smooth bed conditions. The aim of this section is then to verify whether this drag reduction is still observed with a rough bed. The 24 meters downstream-most section of the flume is modified by including rough walls made of aluminium tear plates of typical tears roughness height equal to $1 \mathrm{~mm}$ (see Fig.3). The resulting channel width then reduces to $b_{2}=11 \mathrm{~cm}$.

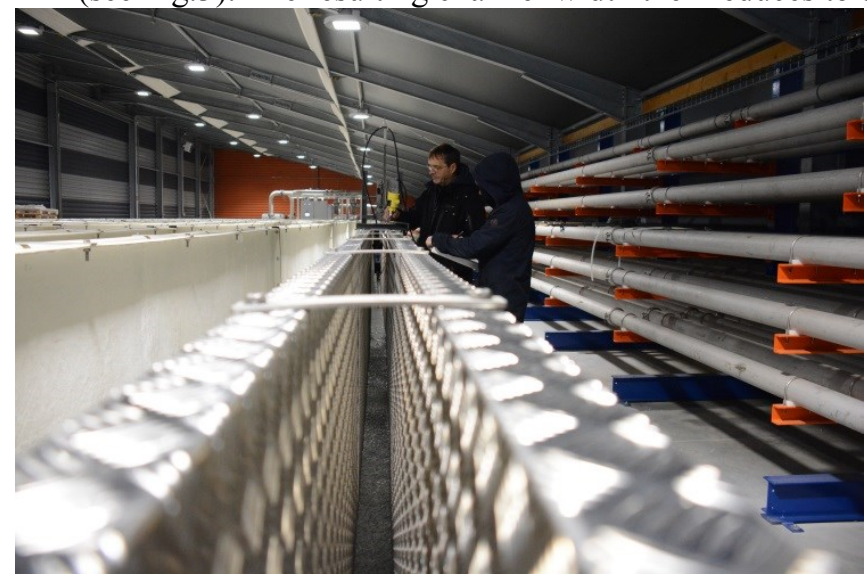

Fig. 3. Photograph of the rough bed channel along with the use of the ultrasonic sensor for the water depth measurement. 
Similar backwater curves, as for the smooth case, are measured and plotted in Fig.4 with a critical depth downstream, for two discharges without additives and with $20 \mathrm{ppm}$ of polymers. As for the smooth bed configuration, the water depths appear to strongly decrease when the polymer is included. When using plain water, the best-fit Darcy coefficient equals $\lambda \sim 0.05$ for both discharges (Fig.4), in agreement with Moody diagram. When adding 20ppm of polymers, the Darcy coefficient reduces to $\lambda \sim 0.037$ for $Q=100 \mathrm{~m}^{3} / \mathrm{h}$ and $\lambda \sim 0.03$ for $Q=50 \mathrm{~m}^{3} / \mathrm{h}$. We thus notice a decrease of Darcy coefficient by a factor 1.35 to 1.66 compared to the configuration with plain water, i.e. a smaller decrease than when using a smooth bed but still a notable drag reduction.

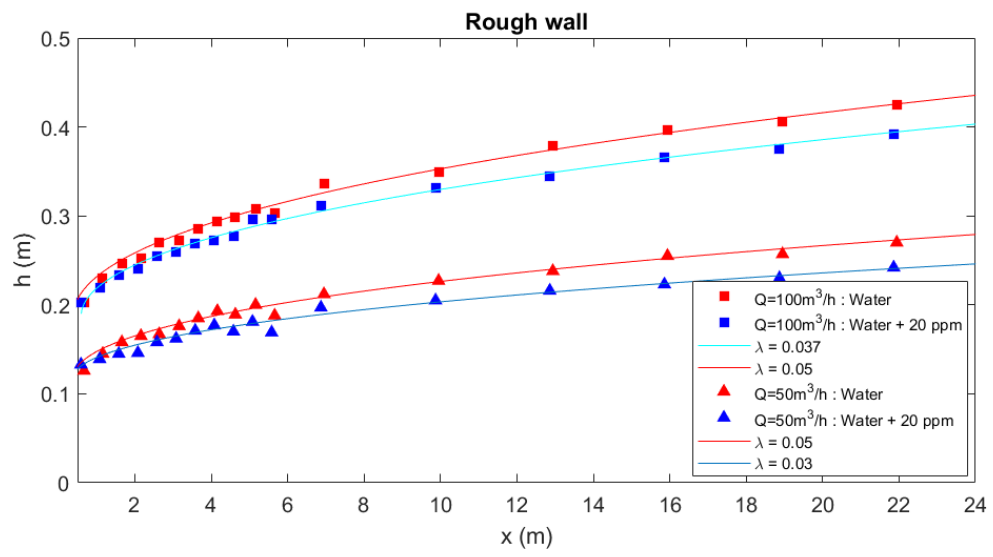

Fig. 4. Backwater curves measured (symbols) and fitted with a constant Darcy friction coefficient (lines) for two discharges $Q=50 \mathrm{~m}^{3} / \mathrm{h}$ and $Q=100 \mathrm{~m}^{3} / \mathrm{h}$ without and with polymers along with the constant fitted Darcy coefficient curves (as for Fig. 1)

Vertical profiles of velocity are also measured in the rough bed configuration in a similar way as for the smooth bed configuration at the same location $(x=6.87 \mathrm{~m})$ for both tested discharges without and with additives; they are shown in Fig. 6 in the same way as for Fig.3. As for the smooth bed condition, very similar streamwise velocity profiles are measured without and with additives over the rough bed for both discharges with a quite constant streamwise velocity $U_{x} / U_{b} \sim 1.2$ for $z / h>0.4$ and a velocity decrease closer to the bed.

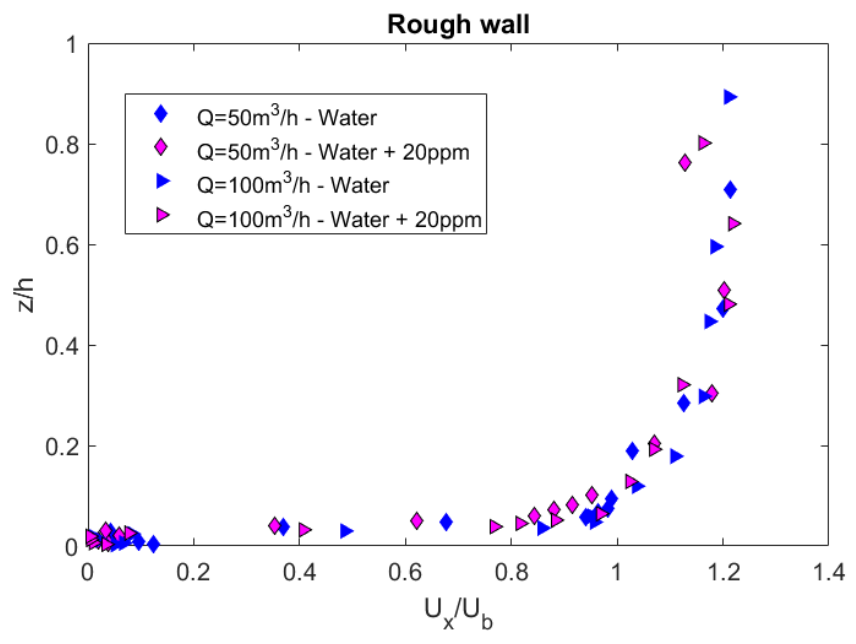


Fig. 5. Vertical profiles of non-dimensional mean streamwise velocities for the two discharges with and without polymers

\section{Discussions and conclusions}

The present paper was dedicated to investigate the impact of adding to the water a limited concentration of polymers for canonical free-surface open-channel flows: 1D subcritical flows over smooth and rough beds. These flow configurations are small-scale and simplified versions of typical channel and river flows. Selected measurements consisted in backwater curves for gradually varied flows and vertical profiles of flow velocities across the water column. These measurements revealed that:

- adding polymers to the water strongly decreases the drag force that the walls apply on the flow, leading to highly increased mean velocities and highly decreased water depths.

- when using a rough wall, the drag reduction is thus still effective but reduced in magnitude compared to a smooth bed configuration, in agreement with previous observations in rough pipes [5]. The averaged Darcy friction coefficient was decreased by a factor of about 2 for the smooth bed configuration and about 1.5 for the rough bed configuration.

- adding polymers to the water hardly modifies the vertical profiles of streamwise dimensionless velocities, at least away from the wall region.

To summarize, the modification of the boundary layer by the additives, detailed in the literature, permits to highly increase the capacity of a watercourse, i.e. to limit the water depths and specifically the overflows over the side banks.

To the author's knowledge, this approach was never tested at large scale in natural freesurface flows such as real rivers and irrigation channels. Whether this method of adding polymers in limited concentrations would be effective at large scale thus remains unknown, but it is highly probable given that it proved to be effective in sewer networks, i.e. at the scale of a narrow watercourse. To prove this feasibility, similar experiments as the ones detailed herein should now be performed in the field, first in small scale canal in highly controlled conditions and then in larger rivers.

A noticeable limitation is related to environmental constraints. River basin managers or regulatory officers may be reluctant to add a chemical component in rivers for mitigating a flood risk. However it must still be kept in mind that i) polyacrylamide by itself does not present any toxicity, only a few ppb in water of residual monomer (acrylamide), which is readily biodegradable, may attract questions ii) polyacrylamide is widely used by highly regulated drinking water treatment plants in developed countries iii) a very low dosage of polymer, 5 to $20 \mathrm{ppm}$, is sufficient for maximum effectiveness while related COD remains lower than $22 \mathrm{mg} / \mathrm{l}$.

All in all, as for any innovation, rivers basin decision makers would have to balance the flood mitigation benefit brought by this new method against its inherent drawback, and make decision accordingly [10].

\section{References}

1. B. A. Toms, Some observation on the flow of linear polymer solutions through straight tubes at large Reynolds number, Proceedings of the First International Congress of Rheology vol. II, The Netherlands, Amsterdam, 135-141 (1949).

2. H. Schlichting, Boundary Layer Theory, McGraw-Hill, New-York, 602-609 (1979).

3. P. S. Virk, AIChE J. 21, 625-656 (1975). 
4. W. H. Cai, F. C.Li, H. N. Zhang, X. B. Li, B. Yu, J. J. Wei, Y. Kawaguchi, K. Hishida, Phys. of Fluids, 21, 115103 (2009).

5. P. S. Virk, J. of Fluid. Mech. 45 (2), 225-246 (1971).

6. J. Hart, L. Cotter, V. Jason, Polymer Addition to Increase Trunk Sewer Flow Capacity at the Resort Municipality of Whistler during the 2010 Winter Olympic Games, Proceedings of the Water Environment Federation, WEFTEC 2011: session 88, 12, 5763-5774 (2011).

7. W. J. Han, Y. Z. Dong, H. J. Choi, Processes 5, 24 (2017).

8. R. I. Tanner, Engineering rheology, Oxford Oxfordshire: Clarendon Press, Oxford Engineering Science Series (1988).

9. Q. Rendu, E. Mignot, N. Riviere, B. Lamberti-Raverot, S. Puijalon, F. Piola, Env. Fluid Mech. 17, 1051 (2017).

10. R. Pich, Method for limiting freshet levels and controlling floods, Patent\# WO 2014199037 A1 (2014). 